\title{
Helvetas: Trend zu partizipativen Technologien
}

Marc Steinlin

\section{(2) OpenEdition \\ Journals}

Electronic version

URL: http://journals.openedition.org/sjep/553

ISSN: 1663-9677

Publisher

Institut de hautes études internationales et du développement

Printed version

Date of publication: 1 novembre 2003

Number of pages: 145-147

ISSN: 1660-5926

Electronic reference

Marc Steinlin, « Helvetas: Trend zu partizipativen Technologien », Schweizerisches Jahrbuch für Entwicklungspolitik [Online], 22-2 | 2003, Online erschienen am: 10 Juni 2010, abgerufen am 07 September 2020. URL : http://journals.openedition.org/sjep/553 


\section{Helvetas: Trend zu partizipativen Technologien}

\section{Marc Steinlin*}

\section{IKT - ein neues Entwicklungsthema}

In ihren Anfängen waren IKT das Tummelfeld von innovativen Pionieren, die auch im Bereich der Entwicklungszusammenarbeit (EZA) neue Technologien und Anwendungen hervorbrachten. Im Verlaufe der 90erJahre hoben zuerst die grossen Akteure (u.a. internationale Organisationen der UNO sowie grosse bilaterale Agenturen wie USAID) das Thema IKT zuoberst auf die Entwicklungsagenden.

Helvetas wurde 1999 durch ein Mandat des Weltbankinstituts mit der Thematik konfrontiert. Sein WorldLINKS for Development-Programm machte augenfällig, welchen Stellenwert diesem Feld eingeräumt wird. Es wurde zudem klar, dass sich hier nicht nur ein zukunftsträchtiges Aktionsfeld eröffnete, sondern dass sich Helvetas den Herausforderungen und Fragen aktiv annehmen musste.

Damit fand sich Helvetas in einem Spannungsfeld wieder. Einerseits erkannte sie die Wichtigkeit dieser neuen Technologien: ein ungeheures Entwicklungspotenzial, neue Lösungsmöglichkeiten für alte Problemstellungen, Wachstumsmöglichkeiten für den Süden wie auch für Helvetas als Organisation. Andererseits bestand eine gewisse Verunsicherung, hervorgerufen durch die Neuartigkeit der Thematik, mangelnde Orientie- rung und Erfahrung, den sich öffnenden digitalen Graben. Befürchtungen, alte Entwicklungsfehler auf neuem Gebiet zu wiederholen (Phänomen der „Rostenden Traktoren“, also „hardware-lastige“ Projektarbeit ohne nachhaltige Prozessentwicklung) wurden wach.

Helvetas reagierte auf diese Herausforderung mit einer Orientierungsphase: Exemplarisch wurde 1999 in Mali eine Feldstudie über IKT verfasst. Daraufhin erarbeitete Helvetas im internen Diskurs erste Orientierungen und Richtlinien und suchte Nischen, die sich ins allgemeine Programm der Organisation einfügten. Daraus resultierte eine eigene Helvetas IKT-Strategie.

\section{Welchen Platz nehmen die IKT in der Helvetas-Strategie ein?}

IKT verändern die EZA-Praxis von zwei Seiten: Sie stellen einerseits neue Herausforderungen an die EZA. Neue Problemstellungen und veränderte Rahmenbedingungen verlangen neue Aktivitätslinien. Andererseits eröffnen sie neue Möglichkeiten, althergebrachte - und oft ungelöste Probleme auf neuen Wegen in Angriff zu nehmen.

Die Bewusstseinsbildung über die globale Informations- und Kommunikationsrevolution hat im Rahmen des Empowerments grundsätzliche Bedeu- 
tung. Die selbstbestimmte, lokale Gestaltung des eigenen Lebens erfordert Kenntnisse, Aufklärung und ein Einschätzungsvermögen dieser globalen Veränderungsprozesse.

In jedem Fall spielt der Zugang zu Informationsressourcen eine Schlüsselrolle. Insbesondere können IKT als „partizipative Technologien“ einen wichtigen Beitrag zum Einbezug marginalisierter Gruppen, welche beispielsweise im Rahmen der Bauernberatung erfolgen kann, leisten. Auch im Kontext von Demokratisierung und Dezentralisierung verfügen IKT künftig über ein grosses Potenzial zur Förderung von Meinungsbildung und Dialog. Nicht zuletzt eröffnen die IKT auch neue Möglichkeiten zum Erfassen und Verbreiten von lokalem Wissen sowie zur Interaktion mit modernem, globalem Wissen, was eine neue Ausprägung des Wissensmanagements erforderlich macht. In diesem Sinn müssen die IKT als vektorielle Komponente in allen Projekten berücksichtigt werden, in denen sie einen Mehrwert versprechen.

Darüber hinaus wurden IKT aber auch als Subsektor der HelvetasArbeit definiert. Sie verlangen spezielle Aufmerksamkeit im Bereich der Bildung. Für Helvetas stellt „Computern“ (die Fähigkeit Computer nutzen zu können) eine vierte Kulturkompetenz (nebst Lesen, Schreiben und Rechnen) dar. IKT werden zu einem integralen Bestandteil der formalen Bildung, was die Aufmerksamkeit auf vier Aspekte lenkt: „Computer Literacy“ (mit Betonung auf Konzeptwissen in IT), IT-Pädagogik, IT-Didaktik sowie die Lehrplanentwicklung. Angesichts der gewaltigen Aufgabe, dem Süden die IKT nahe zu bringen, misst Helvetas aber auch der informellen Bildung einen grossen Stellenwert bei. Sie stützt sich dabei auf intrinsische Entwicklungskräfte, das heisst auf die Entdeckungslust und die autodidaktischen Kräfte innovativer Individuen. Dazu entwickelte Helvetas das Konzept „Infotopes“. Dabei handelt es sich - in Analogie zu Biotopen - um Orte zum Austausch von Wissen und Information, und zur aktiven Auseinandersetzung mit IKT. Computer und das Internet sollen hier der Zielgruppe als Experimentierfeld möglichst frei zugänglich gemacht werden. Zudem sollen entsprechende neue Erschliessungs- und Lernmethoden evaluiert und weiterentwickelt werden.

Generell muss sich die IKT-Strategie bei Helvetas in die bestehenden, übergeordneten Strategien und Prinzipien einfügen. Aus observierten Defiziten und Fehlern anderer Akteure lernend, hat Helvetas für alle ihre Aktivitäten ihr eigenes Leitkonzept der ,four wares" entwickelt. Nebst Hard- und Software definierte sie Frame- und Brainware und legte den Fokus ihrer Aktivitäten auf letztere. Frameware nimmt sich systemischen Fragen zu den IKT an: gesetzliche und soziale Rahmenbedin- gungen, organisatorische und institutionelle Aspekte (Organisational Development), Fragen des Zugangs zu IKT (auch im sozialen Sinn), Unterhaltsprobleme, Wissensmanagement etc. Brainware dagegen umfasst den Bereich Human Resources, Trainingsaspekte und pädagogische Fragen. Nachhaltige Projektarbeit bedingt eine Balance zwischen allen vier Bereichen. 
Was bringt die IKT-Politik von Helvetas in der Praxis?

Anhand von drei Beispielen lässt sich die Strategie illustrieren. Im Rahmen der langjährigen Zusammenarbeit mit den LehrerInnen-Seminaren in Bhutan erarbeitet Helvetas die Einführung der IKT als Ausbildungsfach. Einerseits sollen zukünftige Lehrkräfte IKT für die eigene Lehrtätigkeit nutzen können, andererseits aber bald auch in die Lage kommen, ihrerseits IKT als Fach zu unterrichten. Dazu müssen verschiedene Faktoren erfüllt sein: neben dem nachhaltigen Betrieb der technischen Infrastruktur müssen sich DozentInnen die notwendigen pädagogischen Kenntnisse und Konzepte aneignen. Zudem muss der freie Zugang der StudentInnen zu Computern geöffnet und geregelt werden.

In Paraguay werden IKT als zentrales Mittel in der Bauernberatung eingeführt. BeraterInnen werden mit Notebooks ausgestattet und vernetzt. In der Weiterentwicklung des Wissensmanagements unterstützen sie sich im Feldeinsatz gegenseitig per Inter- net. In Zukunft soll auch die direkte Vernetzung von Bauern zum Zwecke des Austausches lokalen Wissens ermöglicht werden.

Im Rahmen einer Dezentralisierung der staatlichen Administration in Mali sollen regionale Beratungsstellen für Gemeindeverwaltungen per Internet vernetzt werden. Dies ermöglicht den (Wissens-)Austausch mit der zentralen Administration in Bamako, die Verbreitung von Informationen und Ressourcen (z.B. Verwaltungsdokumente), und längerfristig die Einführung von Elementen der „E-Governance“. Dies ist besonders attraktiv, wo auf herkömmlichen Kanälen fast keine Kommunikation und Interaktion möglich ist.

Last but not least haben IKT auch einschneidende Auswirkungen auf die Kommunikation innerhalb Helvetas. Sie ermöglichen mit Hilfe von Intranet das Zusammenrücken ihrer ExpertInnen, und damit eine verbesserte Verfügbarkeit und Nutzbarmachung ihres Fachwissens rund um den Globus. 